\title{
Nasal Septum Warthin's Tumor: A Unique Case Report and Review of the Literature
}

\author{
Andrea LC Carobbio ${ }^{1}$, Giacomo Sollini ${ }^{2}$, Francesco Mazzola ${ }^{3}$, Andrea landelli ${ }^{4}$, Valerio G Vellone ${ }^{5}$, Frank RM Canevari ${ }^{6}$, \\ Giorgio Peretti ${ }^{7}$
}

\begin{abstract}
Warthin's tumor (WT) is the second most common benign salivary gland neoplasm following pleomorphic adenoma, accounting for approximately 5 to $15 \%$ of all salivary gland tumors. Extraparotid localizations of WT are only rarely described in ectopic salivary tissues. Salivary tumor findings are generally uncommon in the nasal cavity and usually correspond to malignant forms. Herein, we present the only case of nasal WT reported in the literature to our knowledge. A 56-year-old woman with a mild and intermittent right epistaxis lasting 1 year came to our attention reporting/ referring slowly growing mass in the right nasal vestibule from 6 months. Personal history showed 40 years of smoking habit. Management and Outcome: Endoscopic evaluation, T1- and T2-weighted magnetic resonance imaging sequences, and subsequent surgical removal with diode laser under general anesthesia were performed. The final histopathological diagnosis was margin-free nasal localization of WT. A PubMed research was performed with the following terms: [(adenolymphoma OR lymphomatous cystadenoma OR cystadenolymphoma OR papillary cystadenoma lymphomatosum OR Warthin) AND (nasal OR sinonasal OR nasal septum)], as long as a cross-linked references research from the articles found. No eligible articles were identified. The existence of extraparotid WT (EPWT) is believed to be a consequence of late encapsulation of the parotid gland during embryologic development with the intermingling of salivary and lymphoid tissues. Other authors have suggested that the lymphocytic component is the result of an immunological reaction to epithelial component proliferation. Nasal tumors are rare and extremely variable. Among salivary neoplasms in the nasal and paranasal sinuses district, pleomorphic adenoma cases were reported, but no nasal WT can be found described in the literature. Despite the unicity of this finding, sharing the diagnostic and therapeutic details of this case was considered to be potentially useful.

Keywords: Benign nasal neoplasm, Cystadenolymphoma, Extraparotid salivary tumor, Warthin's tumor.

Clinical Rhinology An International Journal (2019): 10.5005/jp-journals-10013-1353
\end{abstract}

\section{INTRODUCTION}

Warthin's tumor (WT), also known as papillary cystadenoma lymphomatosum or cystadenolymphoma, is a benign tumor of the salivary glands. WT is the second most common salivary gland neoplasm following pleomorphic adenoma, accounting for approximately 5 to $15 \%$ of all salivary gland tumors. ${ }^{1-3}$ These lesions commonly affect individuals in their sixth to seventh decade of life with a slight male predominance. ${ }^{3}$

WT is almost exclusively restricted to the parotid gland with a preference for the inferior pole. ${ }^{4}$ From 12 to $19 \%$ of patients develop more than one WT, frequently with bilateral parotid involvement (incidence: $5-14 \%) .{ }^{5}$

Extraparotid localizations of WT are only rarely described in ectopic salivary tissues in the latero-cervical and para-parotid lymph nodes, lip, cheek, hard palate, lacrimal gland, larynx, and nasopharynx. ${ }^{6,7}$ Salivary tumor findings are generally rare in the nasal cavity and usually correspond to malignant forms. ${ }^{8}$

Herein, we describe the first reported case in the literature of WT arising from the nasal septum.

\section{Case Description}

A 56-year-old woman was referred to our ENT clinic with a history of mild right epistaxis lasting for 1 year. Furthermore, the patient reported the appearance of a slowly growing mass in the right nasal vestibule from 6 months. The patient reported a 40-year history of smoking (40 pack/year), but no history of alcohol abuse. She had no history of nasal surgery or previous radiation therapy but had
1,3,4,6.7 Department of Otorhinolaryngology and Head and Neck Surgery, IRCCS Ospedale Policlinico San Martino, Genova, Italy ${ }^{2}$ Department of ENT Department, Ospedale Bellaria, Bologna, Italy

${ }^{5}$ Department of Integrated Surgical and Diagnostic Sciences, IRCCS Ospedale Policlinico San Martino, Genova, Italy

Corresponding Author: Andrea LC Carobbio, Department of Otorhinolaryngology and Head and Neck Surgery, IRCCS Ospedale Policlinico San Martino, Genova, Italy, Phone: +39 0105555680, e-mail: a.carobbio@studenti.unibs.it

How to cite this article: Carobbio ALC, Sollini G, Mazzola F, et al. Nasal Septum Warthin's Tumor: A Unique Case Report and Review of the Literature. Clin Rhinol An Int J 2019;12(1):31-34.

Source of support: Nil

Conflict of interest: None

received adenotonsillectomy and appendicectomy in childhood. No oncological history was reported.

Nasal endoscopy revealed a round-shaped mass in the right nasal fossa, about $2 \mathrm{~cm}$ in diameter and covered by normal mucosa (Fig. 1). During diagnostic endoscopy, surgical cottonoids were positioned around the mass in order to detect its attachment, and it appeared in relationship with nasal septum.

Magnetic resonance imaging showed a mass about $2 \mathrm{~cm}$ in diameter in the right nasal vestibule that was hypointense in T1-weighted sequences, hyperintense in T2-weighted sequences with a peduncle in the nasal septum and without local signs of invasion of surrounding structures (Fig. 2). 
Given the benign aspect of the mass, surgical removal under general anesthesia was planned. The mass was removed with a pure endoscopic transnasal approach. In order to obtain the least invasive impact possible, we first displaced the mass by blunt dissection using cottonoids and searching for the pedicle in the septal region. We started removal using a diode laser with incision of the mucosa on the superior border of the lesion. The dissection was continued using a diode laser to separate the mass from nasal vestibule tissue. The insertion point was finally located in the nasal septum, and subperiosteal ablation of septal mucosa was performed. The borders of resection were sent for intraoperative histological examination and were free of disease. Surgical specimens were oriented and sent for definitive histological examination. All specimens were fixed in 10\% formalin, embedded in paraffin blocks, and sections were analyzed following staining with hematoxylin and eosin.

The tumor specimen was extensively sampled and examined with multiple histological slides at different levels. Microscopic examination at low magnification revealed a well-demarcated, rounded epithelial tumor. The lesion was very homogeneous in all slides examined and composed of papillary epithelial proliferation of bilayered oncocytic epithelium growing endophytically into underlying stroma. The papillae had fibrovascular cores with

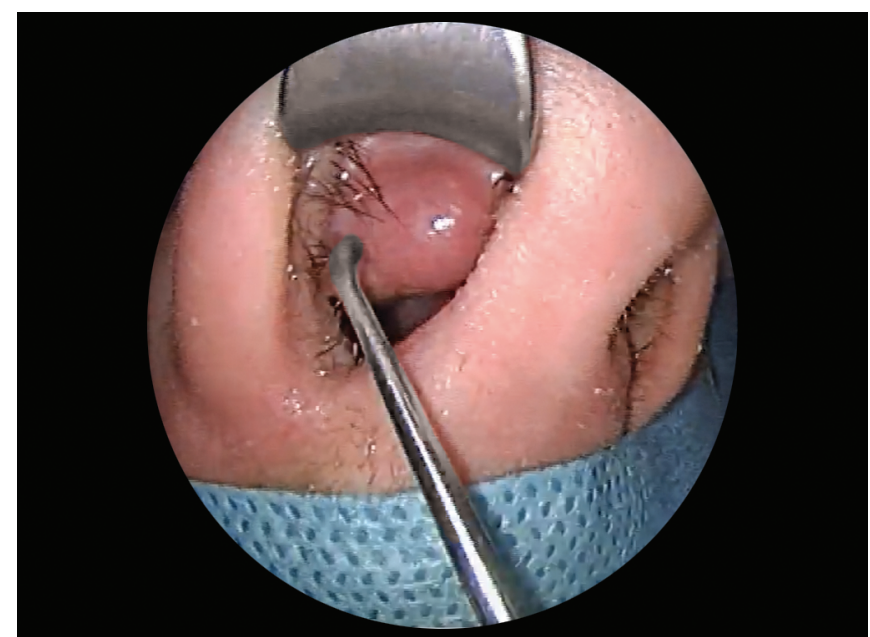

Fig. 1: Preoperative appearance of the lesion in the right nasal vestibule lymphoid stroma. The stroma was characterized by lymphoid tissue with an increased number of mast cells and plasma cells. Mitoses and necrosis were not found nor were areas of different cellular differentiation (Fig. 3). The final histopathological diagnosis was margin-free nasal localization of WT. Due to the uniqueness of this report, we sent tissue specimens for a "second look" pathological evaluation that confirmed the first diagnosis.

The patient underwent regular follow-up visits after surgery. Due to the localization of the mass, outpatient endoscopic examination was considered sufficient to monitor nasal cavity healing and assess for potential recurrence of disease. Furthermore, due to the histology of the tumor, no radiological locoregional follow-up was needed. The last postoperative visit was performed about 2 years after surgery. At that time, no signs of recurrence of nasal WT, local infection, or other postoperative complications were identified. Good healing of the surgical field was found at endoscopic examination, and the site of excision was covered by normal septal mucosa (Fig. 4).

\section{Discussion}

We report the case of a true WT arising from the nasal septum. In order to give more accurate information on this rare case, we performed a literature review without language restriction. We searched articles in PubMed (up to March 2018) with the following terms: [(adenolymphoma OR lymphomatous cystadenoma OR cystadenolymphoma OR papillary cystadenoma lymphomatosum OR Warthin) AND (nasal OR sinonasal OR nasal septum)], and with available cross-linked references from the articles found. No eligible article was identified. To the best of our knowledge, this is the only case of nasal WT reported in the literature to date.

Tumors of minor salivary gland origin occur in the nasal cavity as well as in the paranasal sinuses and much less commonly in the nasopharynx. Their frequency conforms to the density and distribution of the seromucous (salivary gland type) glands of the region and hence their predilection for the nasal (septum and turbinates) and ostial regions.

In a recent and large retrospective study on 1,084 cases of WT, only 10 originated in upper neck, 4 in the submandibular gland, and just 1 in the upper lip. ${ }^{2}$

Salivary-type neoplasms of the nasal cavity and paranasal sinuses are numerically dominated by adenoid cystic carcinomas and pleomorphic adenomas. All others, benign or malignant, are
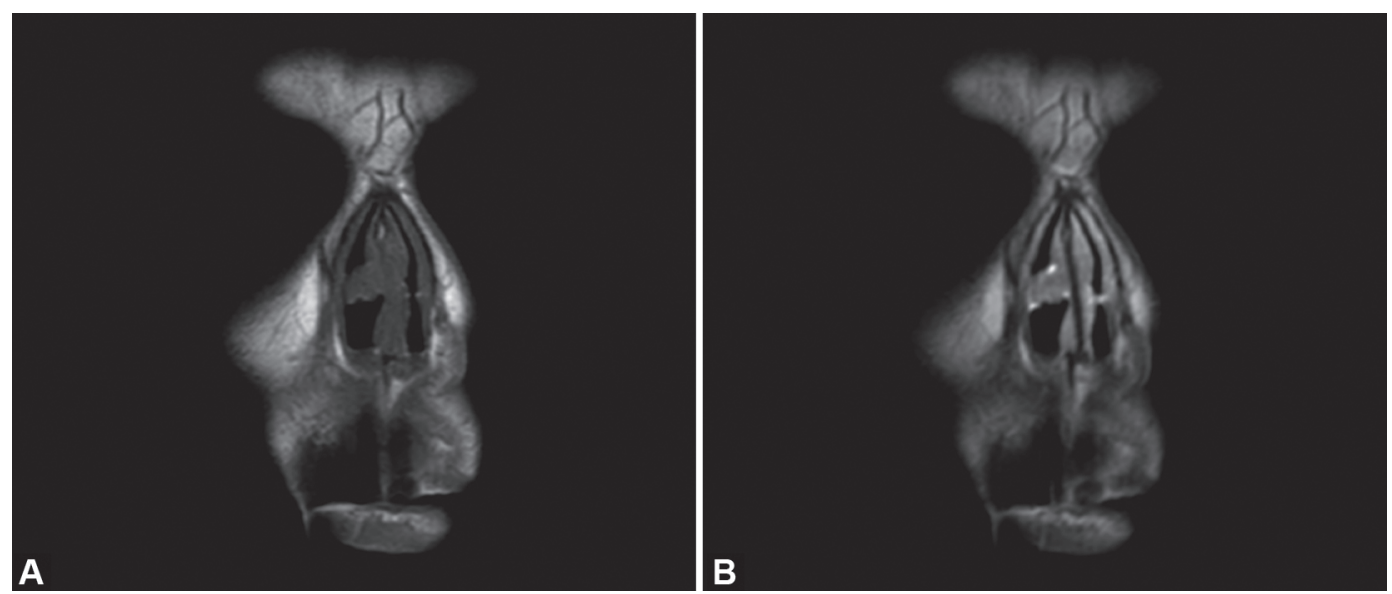

Figs $2 \mathrm{~A}$ and B: Coronal sections of MRIT1- (A) and T2- (B) weighted sequences showing the lesions in the right nasal vestibule and its relationship with the surrounding nasal structures 


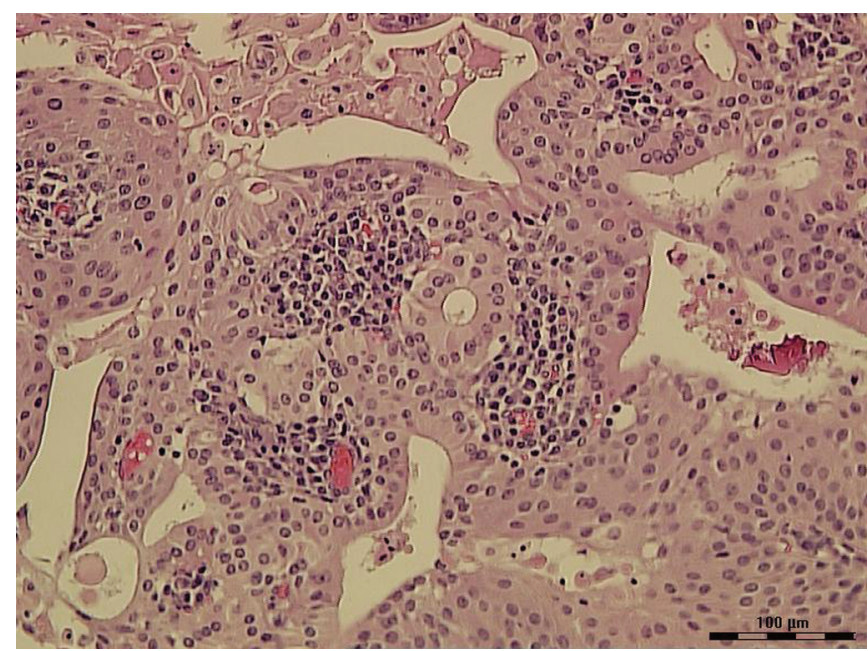

Fig. 3: Hematoxylin/eosin; original magnification $\times 100$ : the tumor shows a papillary and tubular architecture composed of columnar oxyphilic cells with scarce atypia sustained by a lymphocyte-rich stroma. No necrosis, brisk mitotic activity, or signs of destructive invasion were observed

rarely encountered and are usually biologically and histologically low grade. Sites of origin in the sinonasal tract conform to the density and distribution of the seromucous glands and are most often nasal (septum and turbinates) or ostia. ${ }^{9}$

In 1953, Struthers et al. ${ }^{10}$ described a lesion of the maxillary sinus having histologic similarities with WT and defining it as an "atypical" WT due to the paucity of lymphoid stroma. In fact, since the first description by Warthin in 1929, specific histologic features have generally been accepted as mandatory for the diagnosis of cystadenolymphoma. These include irregular cystic spaces usually with amorphous acidophilic material inside; oncocytic epithelial cells lining these papillary cystic structures; and subepithelial stroma of lymphoid tissue.

Snyderman et al. ${ }^{6}$ pointed out that failure to observe this requirement for the histologic components described earlier has led to the erroneous description of WT in unusual extraparotid sites such as the minor salivary glands of the oral cavity and oropharynx, larynx, maxillary sinus, and lacrimal glands. Most extraparotid WT lacks the lymphoid component and so would be more appropriately called oncocytic papillary cystadenomas. They are rare benign tumors, with a clinical presentation similar to WT but usually arise in the minor salivary glands $(0.9-2 \%$ of all minor salivary gland neoplasms) while being extremely rare in the major salivary glands.

Recently, a Warthin-like variant of mucoepidermoid carcinoma often associated with an unusual fusion gene has been described by Ishibashi et al. ${ }^{11}$

Mucoepidermoid carcinoma usually shows a wide morphologic spectrum, including epithelium with oncocytic or squamous metaplastic changes overlying a prominent cystic architecture as well as tumor-associated lymphoid tissue masquerading malignant behavior under the appearance of a benign WT. ${ }^{12}$

In our case, no area of squamoid and goblet "metaplasia" nor atypia or infiltrative aspects were observed, ruling out this differential diagnosis. The histological features of the tumor in our patient fully satisfied the pathological criteria of WT.

The origin of WT is still controversial and has been the subject of much debate. A widespread hypothesis suggests that parotid WT can arise from salivary duct epithelium inclusions in the parotid

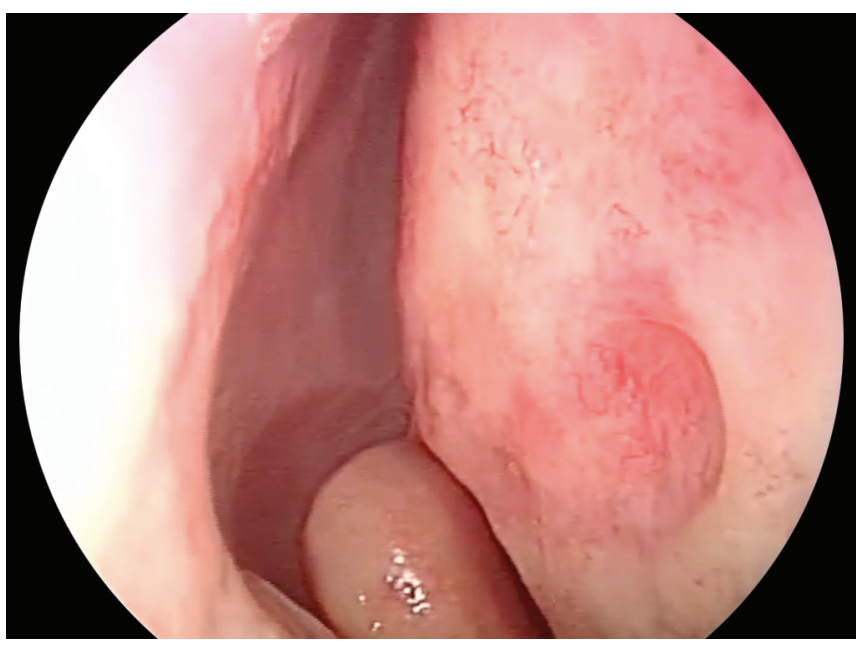

Fig. 4: Postoperative appearance of the surgical field 2 years after the operation

gland lymph nodes, during ontogeny. The existence of extraparotid WT (EPWT) is believed to be a consequence of late encapsulation of the parotid gland during embryologic development with the intermingling of salivary and lymphoid tissues. ${ }^{6}$ Salivary ducts and acini trapped within extraparotid lymph nodes are believed to be the sites of origin of these tumors. The epithelial component of WT is thought to develop from uncommitted duct reserve cells.

Nonetheless, other authors ${ }^{13}$ have proposed that the lymphocytic component is the result of an immunological reaction to epithelial component proliferation or that it may arise as an inflammatory response. This could better explain the very rare extra-parotid localizations such as nasopharynx, larynx, hard palate, or nasal cavity, where lymph nodes are not present but where minor salivary glands can be found.

\section{Conclusion}

Herein, we present the only case of nasal WT reported in the literature to our knowledge. It is worth considering this exceedingly rare histology during the diagnostic pathway, together with all other infrequent nasal tumors. Further studies are still needed, with the help of pathologists, to better focus on all possible uncommon lesions that the ENT specialist may encounter during nasal endoscopy.

\section{References}

1. WHO classification of head and neck tumors - NLM Catalog - NCBI.

2. Xu W, Lu H, Zhu Y, et al. Warthin's tumor in oral and maxillofacial regions: an 18-year retrospective study of 1084 cases in an easternChinese population. Int J Oral Maxillofac Surg 2018;47(7):913-917. DOI: 10.1016/j.ijom.2018.03.013.

3. Tian $Z$, Li L, Wang $L$, et al. Salivary gland neoplasms in oral and maxillofacial regions: a 23-year retrospective study of 6982 cases in an eastern Chinese population. Int J Oral Maxillofac Surg 2010;39(3): 235-242. DOI: 10.1016/j.ijom.2009.10.016.

4. Tartaglione T, Botto A, Sciandra M, et al. Differential diagnosis of parotid gland tumors: which magnetic resonance findings should be taken in account? Acta Otorhinolaryngol Ital 2015;35(5):314-320. DOI: 10.14639/0392-100X-693.

5. Kremp AN, Nelson BL. Bilateral Warthin tumors of the parotid gland. Head Neck Pathol 2008;2(3):175-176. DOI: 10.1007/s12105-0080065-2. 
6. Snyderman C, Johnson JT, Barnes EL. Extraparotid Warthin's tumor. Otolaryngol Head Neck Surg 1986;94(2):169-175. DOI: $10.1177 / 019459988609400207$.

7. Nisa L, Landis BN, Salmina C, et al. Warthin's tumor of the larynx: a very rare case and systematic review of the literature. J Otolaryngol Head Neck Surg 2015;44(1):16. DOI: 10.1186/s40463-0150067-5.

8. Pantvaidya GH, Vaidya AD, Metgudmath R, et al. Minor salivary gland tumors of the sinonasal region: results of a retrospective analysis with review of literature. Head Neck 2012;34(12):1704-1710. DOI: 10.1002/ hed.21988.

9. Manning JT, Batsakis JG. Salivary-type neoplasms of the sinonasal tract. Ann Otol Rhinol Laryngol 1991;100(8):691-694. DOI: 10.1177/000348949110000819.
10. Struthers AM, Williams HL, Parkhill EM. Papillary cystadenoma of the maxillary paranasal sinus (atypical Warthin tumor). AMA Arch Otolaryngol 1954;59(2):241-244. DOI: 10.1001/ archotol.1954.00710050253016.

11. Ishibashi K, Ito Y, Masaki A, et al. Warthin-like mucoepidermoid carcinoma: a combined study of fluorescence in situ hybridization and whole-slide imaging. Am J Surg Pathol 2015;39(11):1479-1487. DOI: $10.1097 /$ PAS.0000000000000507.

12. Heatley N, Harrington KJ, Thway K. Warthin tumor-like mucoepidermoid carcinoma. Int J Surg Pathol 2018;26(1):31-33. DOI: $10.1177 / 1066896917724889$.

13. Kuzenko YV, Romanuk AM, Dyachenko OO, et al. Pathogenesis of Warthin's tumors. Interv Med Appl Sci 2016;8(2):41-48. DOI: 10.1556/1646.8.2016.2.2. 